\title{
Chiral filtering in graphene with coupled valleys
}

\author{
P. L. e S. Lopes ${ }^{1}$, A. H. Castro Neto ${ }^{2}$ and A. O. Caldeira ${ }^{1}$ \\ ${ }^{1}$ Instituto de Física Gleb Wataghin, Universidade Estadual de Campinas, Campinas, SP 13083-970, Brazil and \\ ${ }^{2}$ Graphene Research Centre and Physics Department, \\ National University of Singapore, 2 Science Drive 3, Singapore, 117542
}

\begin{abstract}
We analyze the problem of electronic transmission through semi-infinite and finite regions of graphene which are characterized by different types of connections between the Dirac points. These valley symmetry breaking Hamiltonians might arise from electronic self-interaction mediated by the dielectric environment of distinct parts of the substrate on which the graphene sheet is placed. We show that it is possible to have situations in which we can use these regions to select or filter states of one desired chirality.
\end{abstract}

\section{INTRODUCTION}

Graphene is a truly two-dimensional (2D) crystal with Dirac-like quasi-particles [1, 2]. These charge carriers are the result of the wavefunction interference due to the honeycomb lattice structure and hence they cannot exist outside the many-body system (electrons-plus-lattice). From this perspective, the low energy, long wavelength, Lorentz invariance in this system is an emergent phenomenon and, as such, can be influenced by external factors such as disorder, applied electric and magnetic fields, and structural deformations of the lattice such as pressure, strain, and shear [3].

The low energy physics of these Dirac quasi-particles is strongly influenced by the fact that the two Dirac cones sit at the corners of the hexagonal Brillouin zone ( $\mathrm{K}$ and $\left.\mathrm{K}^{\prime}\right)$. These cones are related by time reversal symmetry and define the chirality of these quasi-particles in terms of their momentum relative to each cone. Unlike the case of neutrinos, where only one chiral flavor exists, Dirac particles in graphene have both flavors, and they can be either right-handed or left-handed depending on whether they reside in one cone or the other. While in a perfect, non-interacting, graphene sheet these chiral states are decoupled, in a real graphene sample they can be coupled by the external perturbations mentioned above. At low energies, one can have either intra-valley processes, with small momentum transfer, that preserve chirality, or inter-valley processes, with large momentum transfer, that mixes chiralities. It is well-known that, in the presence of weak disorder, intra-valley processes lead to weak anti-localization effects [4, whereas inter-valley processes lead to weak localization [5]. Hence, the coupling between $\mathrm{K}$ and $\mathrm{K}^{\prime}$ points play an important role in the physics of graphene.

In this work we study the transport through regions where the $\mathrm{K}$ and $\mathrm{K}$ ' points are not coupled and regions in which they are actually coupled. Although the Hamiltonians with which we deal are manifestly non interacting, these couplings might arise from the interactions between the electrons, as we argue below. Our main goal is to understand how the Dirac electrons behave across the interfaces separating those two regions as a way to classify the possible scattering mechanisms in graphene. Such a situation can also be artificially created by depositing graphene across substrates with different dielectric constants. For instance, there are quantum Monte Carlo [7] calculations that indicate that suspended graphene (i.e., on vacuum with dielectric constant $\epsilon_{0}=1$ ) is a excitonic insulator and graphene on $\mathrm{SiO}_{2}\left(\epsilon_{\mathrm{SiO} 2} \approx 3\right)$ should be a semi-metal [8]. An interface between those regions would have a transistor-like effect with a large on-off ratio for current flow.

Our starting point is the well-known low energy effective Hamiltonian for neutral graphene that is given by [1] (we use units such that $v_{F}=\hbar=1$ ):

$$
\mathcal{H}=\int d^{2} r\left(\begin{array}{ll}
\hat{\Psi}_{1}^{\dagger} & \hat{\Psi}_{2}^{\dagger}
\end{array}\right)\left(\begin{array}{cc}
\sigma \cdot \hat{\mathbf{P}} & 0 \\
0 & \sigma^{*} \cdot \hat{\mathbf{P}}
\end{array}\right)\left(\begin{array}{l}
\hat{\Psi}_{1} \\
\hat{\Psi}_{2}
\end{array}\right),
$$

where $\hat{\Psi}_{i}^{\dagger}=\left(a_{i}^{\dagger}, b_{i}^{\dagger}\right), a_{i}^{\dagger}$ and $b_{i}^{\dagger}$ being the creation operators for electrons in sublattice $\mathrm{A}$ or $\mathrm{B}$, respectively, in the Dirac cone $i(i=1,2)$ and $\sigma=\left(\sigma_{x}, \sigma_{y}\right)$ is a 2D vector whose components are Pauli matrices (we ignore spin variables).

In the representation of the Hamiltonian (1) there are many ways to couple the $\mathrm{K}$ and $\mathrm{K}$ ' points [8]. These couplings have different symmetries and hence represent different physical processes. Notice, however, that all the processes that couple the two cones can be represented in terms of combinations of the identity matrix and Pauli matrices in the "valley" space. We will deal only with real coupling potentials in such a way that we can write explicitly all the generators of the different couplings as combinations of basic matrices:

$$
\begin{gathered}
\mathcal{V}_{x}=\Delta\left(\begin{array}{cc}
0 & \sigma_{x} \\
\sigma_{x} & 0
\end{array}\right), \\
\mathcal{V}_{y}=\Delta\left(\begin{array}{cc}
0 & -i \sigma_{y} \\
i \sigma_{y} & 0
\end{array}\right), \\
\mathcal{V}_{z}=\Delta\left(\begin{array}{cc}
0 & \sigma_{z} \\
\sigma_{z} & 0
\end{array}\right),
\end{gathered}
$$

and

$$
\mathcal{V}_{I}=\Delta\left(\begin{array}{ll}
0 & 1 \\
1 & 0
\end{array}\right)
$$


where $\Delta$ is a parameter that is assumed to be positive throughout the paper since negative deltas will bring no new physics. Of course the most arbitrary potential would be a linear combination of these with different delta parameters (and in this case the sign difference between them might be relevant).

As we are going to show, only $\mathcal{V}_{y}$ and $\mathcal{V}_{x}$ are of real interest and will be the focus of our studies. We will also show that the transmission through regions described by these matrices presents very unusual properties and can presumably be measured experimentally (we stress, nevertheless, that it is not our aim to fully solve the transport problem considering finite size and disorder effects, but to analyze the physics of our proposed model which we believe can account for the main properties behind, for instance, the contact resistance of nanoscopic graphene junctions).

The paper is organized as follows. In Sec. II we give a very brief introduction to the problem of "free" graphene and some of our notation. In Sec. III we present and analyze the Hamiltonians with which we work, giving a somewhat general notion on why our choices of interactions and their nature, solving the corresponding eigenvalue problems and analyzing the symmetries they obey. In Sec. IV, we (less) briefly discuss the transmission problem in two dimensions. In Sec. V we solve the problem of transmission through the interface between two semiinfinite regions of graphene, one with "free" electrons and the other with self-interacting quasi-particles and finally, in Sec. VI, we solve the finite-barrier-like problem of transmission through a finite region of the interacting material. In Sec. VII, we try to make a connection of our results and the Landauer formalism. In Sec. VIII we present our conclusions.

\section{BASIC PROPERTIES}

The eigenvalue problem given by (1) can be solved by going into the momentum representation

$$
\left(\begin{array}{cc}
\sigma \cdot \mathbf{k} & 0 \\
0 & \sigma^{*} \cdot \mathbf{k}
\end{array}\right) \psi_{ \pm \mathbf{K}\left(\mathbf{K}^{\prime}\right)}(\mathbf{k})=E_{ \pm} \psi_{ \pm \mathbf{K}\left(\mathbf{K}^{\prime}\right)}(\mathbf{k})
$$

with energy eigenvalues, $E= \pm k$, and eigenstates of well defined momenta around the Dirac points (with their corresponding spinors, see below). Going back to the position representation one has $\psi_{\mathbf{K}\left(\mathbf{K}^{\prime}\right)}(\mathbf{r})=\psi_{ \pm \mathbf{K}\left(\mathbf{K}^{\prime}\right)}(\mathbf{k}) e^{i \mathbf{k} \cdot \mathbf{r}}$ where the spinors are

$$
\begin{gathered}
\psi_{ \pm \mathbf{K}}(\mathbf{k})=\frac{1}{\sqrt{2}}\left(\begin{array}{c}
e^{-i \theta / 2} \\
\pm e^{i \theta / 2} \\
0 \\
0
\end{array}\right) \\
\psi_{ \pm \mathbf{K}^{\prime}}(\mathbf{k})=\frac{1}{\sqrt{2}}\left(\begin{array}{c}
0 \\
0 \\
e^{i \theta / 2} \\
\pm e^{-i \theta / 2}
\end{array}\right)
\end{gathered}
$$

with $\theta=\arctan \left(k_{y} / k_{x}\right)$ and the vector $\mathbf{k}$, whose modulus is connected to the Fermi energy by the dispersion relation, is centered either around $\mathbf{K}$ or $\mathbf{K}^{\prime}$, respectively. These eigenstates, which are symmetric and antisymmetric linear combinations of the states referring to the A and B sublattices, allow us to introduce a particlehole representation which reads

$$
\mathcal{H}=\sum_{i=1,2} k\left[f_{k, i}^{\dagger} f_{k, i}-h_{k, i}^{\dagger} h_{k, i}\right]
$$

where the hole states have negative energy. Thus, we describe the electron behavior in the free graphene by a theory of non-interacting massless fermionic quasi-particles with two different "flavors" (and their corresponding anti-particles).

\section{INTERACTING HAMILTONIANS}

Electron-electron interaction plays an important role in graphene physics and transport problems [10, 11]. Its effects depend strongly on the dielectric function and is independent of the electronic density [10]. Since the dielectric function can be affected in many ways, as, for instance, by adsorbed atoms on the surface or deposition substrates, we propose that there may be situations in which the resulting effective electron-electron interaction can cause mixing of the Dirac cones and we show how it could happen, at least in a heuristic mean-field approximation. Our approach is phenomenological, and, therefore, the specific shape of the interaction potentials will not be given and should be chosen from microscopic arguments. There are many possible situations, and one of them is the possibility that the (Coulomb) interaction becomes screened [10, 12. Hence, we justify the choice of our approach and will make the symmetry assumptions we find reasonable to achieve our results.

So, let us suppose that the electron-electron interaction contribution to the usual tight binding Hamiltonian is of the form

$$
H_{I}=\sum_{i \neq j} U_{i j} n_{i} n_{j}
$$

Separating the terms in the summation corresponding to each sublattice, we arrive at

$$
\begin{aligned}
H_{I A} & =\sum_{i \neq j} U_{i j} n_{A i} n_{A j} \\
H_{I B} & =\sum_{i \neq j} U_{i j} n_{B i} n_{B j} \\
H_{I A B} & =\sum_{i, j} V_{i j} n_{A i} n_{B j} .
\end{aligned}
$$

We have assumed that the interaction between the electrons within each sublattice is equal, which actually need not be. 
Now we perform the mean field approximation. The values of the mean-fields should actually be determined in a self-consistent way, which will not be done here, and be justified by microscopic arguments. Our point is that if there is any physical process which gives rise to these mean-fields, the proposed decoupling will lead to our results. We make more comments on the physical nature of this approximation further below. That being said, we can choose, for example, the first Hamiltonian and get, to first order in the deviations from a mean density of electrons in sublattice A,

$$
\begin{aligned}
H_{I A} & \approx \sum_{i \neq j} U_{i j}\left[n_{A i}\left\langle n_{A j}\right\rangle+n_{A j}\left\langle n_{A i}\right\rangle-\left\langle n_{A i}\right\rangle\left\langle n_{A j}\right\rangle\right] \\
& =\sum_{i} \Lambda_{i} n_{A i}-\text { const. }
\end{aligned}
$$

where $\Lambda$ is the effective on-site potential generated by the self-interaction between the particles. In Fourier space, we can write

$$
a_{i}=\frac{1}{\sqrt{N_{S}}} \sum_{\mathbf{k}} e^{i \mathbf{k} \cdot \mathbf{R}_{i}} a_{\mathbf{k}}
$$

where $N_{S}$ is the number of sites on the lattice. Hence, we have,

$$
H_{I A} \approx \sum_{\mathbf{k k}^{\prime}} \Delta_{\mathbf{k}-\mathbf{k}^{\prime}} a_{\mathbf{k}}^{\dagger} a_{\mathbf{k}^{\prime}}
$$

where we have ignored the constant term. Therefore, this Hamiltonian couples the $\mathbf{k}$ vectors. If we keep only the the long wavelength contribution to the expression just above, we find that this term leads to the four couplings (following our notation, $a_{\mathbf{K}} \rightarrow a_{1}, a_{\mathbf{K}^{\prime}} \rightarrow a_{2}$ )

$$
a_{1}^{\dagger} a_{1} ; a_{2}^{\dagger} a_{2}
$$

and

$$
a_{1}^{\dagger} a_{2} ; a_{2}^{\dagger} a_{1} .
$$

The first two lead to diagonal terms which are of no interest to us. The two other give contributions exactly of the type we want. The same arguments can be carried over to $H_{I B}$. Since the potential must be such that $U_{i j}=U_{j i}$, its Fourier transform is real. The mean fields should also be real and this justifies our choice of only analyzing the four real $\mathcal{V}$ Hamiltonians. As for the physical realization of these mean fields, we believe that the presence of the substrate might, in specific cases, affect the electrons in the material and break the symmetries between the sub-lattices in such a way that ordered states like, for example, a CDW could develop. More thought in this direction is needed but will not be pursued in this paper.

If one wants a more thorough approach, one can use the Alicea and Fisher model [13. There it is shown how the long and short range interactions give rise to terms like staggered densities in each sublattice,

$$
\rho_{\text {stag }}=n_{A 1}+n_{A 2}-n_{B 1}-n_{B 2},
$$

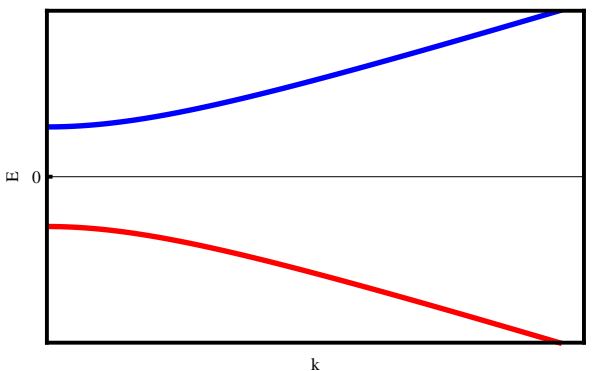

Figure 1: (color on-line) Schematics of the dispersion relation for the Hamiltonian in the presence of the $V_{y}$ term (arbitrary units).

where $n_{A, B i}, i=1,2$, refers to the number operator in the sublattice A or B and Dirac cone 1 or 2 . In the Alicea and Fisher model, the long wavelength Hamiltonian has (local) contributions due to electronic densities given by

$$
\begin{aligned}
\rho_{\text {tot }}^{2}+\rho_{\text {stag }}^{2}= & a_{1}^{\dagger} a_{1} a_{1}^{\dagger} a_{1}+a_{2}^{\dagger} a_{2} a_{2}^{\dagger} a_{2}+ \\
& +b_{1}^{\dagger} b_{1} b_{1}^{\dagger} b_{1}+b_{2}^{\dagger} b_{2} b_{2}^{\dagger} b_{2}+b_{1}^{\dagger} b_{1} b_{2}^{\dagger} b_{2}+ \\
& +a_{2}^{\dagger} a_{2} a_{1}^{\dagger} a_{1}+a_{1}^{\dagger} a_{1} a_{2}^{\dagger} a_{2}+b_{2}^{\dagger} b_{2} b_{1}^{\dagger} b_{1} .
\end{aligned}
$$

This term comes from the on-site repulsion interaction (a more detailed analisys can be found in the original paper [13]). We can see that a mean-field decoupling might also generate our Hamiltonians in this case, however, the mean-fields in this case result directly from the replacement $a_{1} a_{2}^{\dagger} \rightarrow\left\langle a_{1} a_{2}^{\dagger}\right\rangle$, for example. We find this type of mean-field harder to justify physically but leave it here as an example.

As we had formerly proposed, we have given some heuristic arguments for our ad hoc choice of the form of the $\mathcal{V}_{i}$ interactions. We proceed now to exploit the consequences of these model Hamiltonians in some specific cases.

\section{Spectrum in the presence of $\mathcal{V}_{y}$}

In the presence of the disturbance (3) the Hamiltonian is given by

$$
H=\left(\begin{array}{cccc}
0 & k e^{-i \theta} & 0 & -\Delta \\
k e^{i \theta} & 0 & \Delta & 0 \\
0 & \Delta & 0 & k e^{i \theta} \\
-\Delta & 0 & k e^{-i \theta} & 0
\end{array}\right)
$$

The solution of the eigenvalue problem gives $E_{A, B \pm}=$ $\pm \sqrt{k^{2}+\Delta^{2}} \equiv \pm \epsilon$ (Fig. 1) with a gap of size $\Delta$ in the spectrum. The spinorial part of the eigenvectors (we omit 
the plane waves for simplicity in this whole section)

$$
\begin{aligned}
& \left|\chi_{A}^{+}\right\rangle=\frac{1}{\sqrt{2}}\left(\begin{array}{c}
A_{+} e^{-i \theta / 2} \\
A_{+} e^{i \theta / 2} \\
A_{-} e^{i \theta / 2} \\
-A_{-} e^{-i \theta / 2}
\end{array}\right),\left|\chi_{A}^{-}\right\rangle=\frac{1}{\sqrt{2}}\left(\begin{array}{c}
A_{-} e^{-i \theta / 2} \\
A_{-} e^{i \theta / 2} \\
-A_{+} e^{i \theta / 2} \\
A_{+} e^{-i \theta / 2}
\end{array}\right), \\
& \left|\chi_{B}^{+}\right\rangle=\frac{1}{\sqrt{2}}\left(\begin{array}{c}
A_{-} e^{-i \theta / 2} \\
-A_{-} e^{i \theta / 2} \\
-A_{+} e^{i \theta / 2} \\
-A_{+} e^{-i \theta / 2}
\end{array}\right),\left|\chi_{B}^{-}\right\rangle=\frac{1}{\sqrt{2}}\left(\begin{array}{c}
A_{+} e^{-i \theta / 2} \\
-A_{+} e^{i \theta / 2} \\
A_{-} e^{i \theta / 2} \\
A_{-} e^{-i \theta / 2}
\end{array}\right),
\end{aligned}
$$

where $A_{ \pm}=\sqrt{\frac{\epsilon \pm k}{2 \epsilon}}$. Here, $A$ and $B$ do not refer to the different triangular sub-lattices but to the different degenerate states originating from the dispersion relation.

as we saw, can use eq.(7) to put this Hamiltonian in a particle-hole representation, where particle and hole states are described as symmetric and anti-symmetric combinations of the different sub-lattices wave functions. The effective interaction between the particles and holes is then written as

$$
H_{p h}=\left(\begin{array}{cccc}
k & 0 & 0 & \Delta \\
0 & -k & -\Delta & 0 \\
0 & -\Delta & k & 0 \\
\Delta & 0 & 0 & -k
\end{array}\right)
$$

where $p h$ refers to the particle-hole representation. Eq. (20) can be seen to obey orthogonal time reversal symmetry (exchanging the valleys by time reversal) and it is seen to generate a band gap. We see that this kind of effective potential is equivalent to the introduction of an asymmetric coupling between particles and holes from different Dirac cones.

\section{Spectrum in the presence of $\mathcal{V}_{x}$}

In the presence of a perturbation of the form (2), the full Hamiltonian becomes :

$$
H=\left(\begin{array}{cccc}
0 & k e^{-i \theta} & 0 & \Delta \\
k e^{i \theta} & 0 & \Delta & 0 \\
0 & \Delta & 0 & k e^{i \theta} \\
\Delta & 0 & k e^{-i \theta} & 0
\end{array}\right)
$$

Diagonalization will lead us to $E_{ \pm \pm}= \pm k \pm \Delta$ (Fig. 2) with no gap in the spectrum but a shift of the Dirac cones relative to each other by $\Delta$. The eigenvectors are now

$$
\left|\chi_{++}\right\rangle=\frac{1}{2}\left(\begin{array}{c}
e^{-i \theta / 2} \\
e^{i \theta / 2} \\
e^{i \theta / 2} \\
e^{-i \theta / 2}
\end{array}\right),\left|\chi_{+-}\right\rangle=\frac{1}{2}\left(\begin{array}{c}
e^{-i \theta / 2} \\
e^{i \theta / 2} \\
-e^{i \theta / 2} \\
-e^{-i \theta / 2}
\end{array}\right)
$$

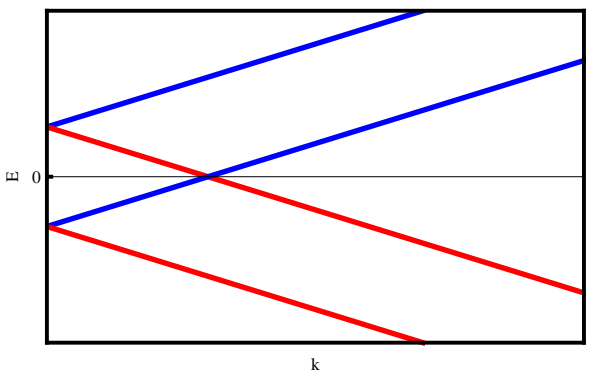

Figure 2: (color on-line) Schematics of the dispersion relation for the Hamiltonian in the presence of the $V_{x}$ term(arbitrary units).

$$
\left|\chi_{-+}\right\rangle=\frac{1}{2}\left(\begin{array}{c}
e^{-i \theta / 2} \\
-e^{i \theta / 2} \\
-e^{i \theta / 2} \\
e^{-i \theta / 2}
\end{array}\right),\left|\chi_{--}\right\rangle=\frac{1}{2}\left(\begin{array}{c}
-e^{-i \theta / 2} \\
e^{i \theta / 2} \\
-e^{i \theta / 2} \\
e^{-i \theta / 2}
\end{array}\right)
$$

and, in the particle-hole representation we have

$$
H_{p h}=\left(\begin{array}{cccc}
k & 0 & \Delta & 0 \\
0 & -k & 0 & -\Delta \\
\Delta & 0 & k & 0 \\
0 & -\Delta & 0 & -k
\end{array}\right)
$$

The Hamiltonian now obeys $\mathcal{S}$ time reversal symmetry and there is no energy gap ( $\mathcal{S}$ is the sympletic time reversal symmetry in the sense described in [17]). However, there is another interesting property that shows up. Because of the different translations in energy of the two Dirac cones, there can be phenomena akin to the Klein paradox in transmission problems. The particle-hole representation reveals an effective interaction that favors the coupling of different valley particles over the coupling of different valley holes states.

\section{Spectrum in the presence of $\mathcal{V}_{I}$ and $\mathcal{V}_{z}$}

Two other Hamiltonians that possibly connect valleys are given by (4) and (5) and therefore,

$$
H=\left(\begin{array}{cccc}
0 & k e^{-i \theta} & \Delta & 0 \\
k e^{i \theta} & 0 & 0 & \Delta \\
\Delta & 0 & 0 & k e^{i \theta} \\
0 & \Delta & k e^{-i \theta} & 0
\end{array}\right)
$$

and

$$
H=\left(\begin{array}{cccc}
0 & k e^{-i \theta} & \Delta & 0 \\
k e^{i \theta} & 0 & 0 & -\Delta \\
\Delta & 0 & 0 & k e^{i \theta} \\
0 & -\Delta & k e^{-i \theta} & 0
\end{array}\right)
$$


These are of no actual interest to transmission problems. The dispersion relations will be, respectively,

$$
E_{ \pm \pm x}= \pm \sqrt{k^{2}+\Delta^{2} \pm 2 k \Delta \cos \theta}
$$

and

$$
E_{ \pm \pm y}= \pm \sqrt{k^{2}+\Delta^{2} \pm 2 k \Delta \sin \theta}
$$

These problems are related to each other by rotations of $\pi / 2$ around the $z$ axis, and hence do not introduce any new physics. Besides, we see that the effect of this potential on the dispersion relations of the particles reduces to just a change by $\pm \Delta$ in the $x$ (first case) or $y$ (second case) components of the particles' momenta, which can be renormalized and the resulting quasi-particles behave no differently from free particles. Therefore, in the regions affected by these potentials, the transmission probabilities will be equal to one, as can be shown by solving the semi-infinite or finite interacting regions problem. We will not deal with these two cases any further and show, only for completeness, the expressions for the spinors associated with the Hamiltonians above, which respectively read:

$$
\begin{aligned}
& \left|\chi_{-+}\right\rangle=\frac{1}{2}\left(\begin{array}{c}
1 \\
-\frac{E_{++x}}{k e^{-i \theta}+\Delta} \\
-\frac{E_{++x}}{k e^{-i \theta}+\Delta} \\
1
\end{array}\right)\left|\chi_{++}\right\rangle=\frac{1}{2}\left(\begin{array}{c}
1 \\
\frac{E_{++x}}{k e^{-i \theta}+\Delta} \\
\frac{E_{++x}}{k e^{-i \theta}+\Delta} \\
1
\end{array}\right)\left|\chi_{+-}\right\rangle=\frac{1}{2}\left(\begin{array}{c}
-1 \\
-\frac{E_{+-x}}{k e^{-i \theta}-\Delta} \\
\frac{E_{+-x}}{k e^{-i \theta}-\Delta} \\
1
\end{array}\right)\left|\chi_{---}\right\rangle=\frac{1}{2}\left(\begin{array}{c}
-1 \\
\frac{E_{+-x}}{k k^{-i \theta}-\Delta} \\
-\frac{E_{+-x}}{k e^{-i \theta}-\Delta} \\
1
\end{array}\right) ; \\
& \left|\chi_{-+}\right\rangle=\frac{1}{2}\left(\begin{array}{c}
1 \\
-\frac{E_{++y}}{k e^{-i \theta}+\Delta} \\
-\frac{E_{++y}}{k e^{-i \theta}+\Delta} \\
1
\end{array}\right)\left|\chi_{++}\right\rangle=\frac{1}{2}\left(\begin{array}{c}
1 \\
\frac{E_{++y}}{k e^{-i \theta}+\Delta} \\
\frac{E_{++y}}{k e^{-i \theta}+\Delta} \\
1
\end{array}\right)\left|\chi_{+-}\right\rangle=\frac{1}{2}\left(\begin{array}{c}
-1 \\
-\frac{E_{+-y}}{k e^{-i \theta}-\Delta} \\
\frac{E_{+-y}}{k e^{-i \theta}-\Delta} \\
1
\end{array}\right)\left|\chi_{--}\right\rangle=\frac{1}{2}\left(\begin{array}{c}
-1 \\
\frac{E_{+-y}}{k e^{-i \theta}-\Delta} \\
-\frac{E_{+-y}}{k e^{-i \theta}-\Delta} \\
1
\end{array}\right) .
\end{aligned}
$$

\section{THE TRANSMISSION PROBLEM IN 2 D. "BARRIERS" AND "STEPS"}

From now onwards, we will be dealing with situations in which there is transmission of electronic waves from "free" electron graphene into semi-infinite or finite regions of "disturbed" graphene, and also reflection back into the original region. We call these two situations step and barrier problems, respectively.

Although there has been extensive use of wavefunction matching to describe problems of electronic behavior in graphene (some examples can be seen in [18] and [19]), we would like to make some comments of our own. We shall start by briefly talking about the simple $2 \mathrm{D}$ scattering from straight interfaces. Although it is a simple problem it is very useful to establish the terminology we employ in the more complex cases. All problems of transmission begin with the determination of the wave functions in different regions and the enforcement of the boundary conditions they obey. We assume that the particle in medium I has positive energy, with momentum on the Dirac cone $\mathbf{K}$ and moves to the right. Since both types of interactions connect states of the different Dirac cones, conservation of the valley "flavor" need not take place any more and the states corresponding to medium I must be given by

$$
\Psi_{I}=\left[\frac{1}{\sqrt{2}}\left(\begin{array}{c}
e^{-i \theta / 2} \\
e^{i \theta / 2} \\
0 \\
0
\end{array}\right)+\frac{r_{1}}{\sqrt{2}}\left(\begin{array}{c}
-i e^{i \theta / 2} \\
i e^{-i \theta / 2} \\
0 \\
0
\end{array}\right)+\frac{r_{2}}{\sqrt{2}}\left(\begin{array}{c}
0 \\
0 \\
i e^{-i \theta / 2} \\
-i e^{i \theta / 2}
\end{array}\right)\right] e^{i \mathbf{k} \cdot \mathbf{r}}
$$

Moreover, if the problem is of a barrier type, we should be able to find in medium III the particles with momenta around both valleys, independently of its flavor in medium I. Conservation of energy demands that these particles in medium III must also have positive energy 
and then we have

$$
\Psi_{I I I}=\left[\frac{t_{1}}{\sqrt{2}}\left(\begin{array}{c}
e^{-i \theta / 2} \\
e^{i \theta / 2} \\
0 \\
0
\end{array}\right)+\frac{t_{2}}{\sqrt{2}}\left(\begin{array}{c}
0 \\
0 \\
e^{i \theta / 2} \\
e^{-i \theta / 2}
\end{array}\right)\right] e^{i \mathbf{k} \cdot \mathbf{r}}
$$

The states accessible to the particles in medium II depend on the dispersion relations of the Hamiltonian and we will deal with each specific case in the next sections.

Since the Hamiltonians are of first order in $\hat{\mathbf{P}}$, we need only to invoke the continuity of the wavefunctions at the interfaces of the different media to be able to determine the coefficients $r_{i}$ and $t_{i}$. However, some care must be taken when evaluating the transmission and reflection amplitudes in the step problems. These must be determined by the conservation of the probability current in the direction normal to the interface (in our case, the $x$ direction). Beginning with the continuity equation we have, for medium $i=\mathrm{I}$, II, III,

$$
\nabla \cdot \mathbf{J}_{i}=-\frac{\partial \rho_{i}}{\partial t}
$$

which in the stationary regime implies that $\rho_{i}=\left|\Psi_{i}\right|^{2}$ is time independent and we have

$$
\nabla \cdot \mathbf{J}_{\mathbf{i}}=0
$$

Now, since our problem is translational invariant along the $y$ direction, $\mathbf{J}_{i}$ is independent of the $y$ variable and it finally reduces to a one dimensional conservation problem

$$
J_{i x}=\text { const. }
$$

Although the conservation of the probability current is relevant only in one dimension, the coefficients $r_{i}$ and $t_{i}$ and the eigenstates (with which we will calculate the probability current) all depend on the angles that the momenta of the particles make with the normal to the interfaces in each medium. Since the states are equal to each other at the interfaces (boundary conditions), we have that

$$
J_{I x}=J_{I I x}=J_{I I I x}
$$

the last equality happening only for barrier problems.

We calculate the probability current in each medium by taking the mean values of the current operator defined by

$$
\mathbf{J}=-\frac{\delta H}{\delta \mathbf{A}}=e \vec{\sigma}
$$

with the states $\Psi_{i}$, where $\mathbf{A}$ is the electromagnetic vector potential. Notice that depending on the type of interaction (for instance, if it is momentum dependent), the expression for the current will not assume this usual simple form and the definition through the functional derivative

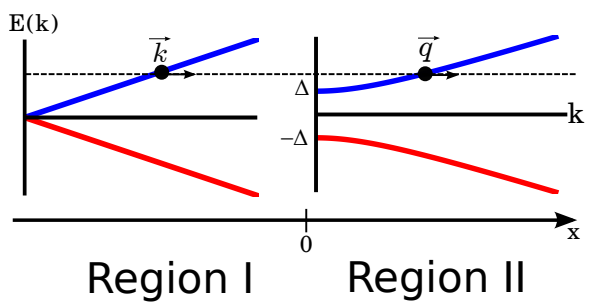

Figure 3: (color on-line) Schematics of the step transmission problem for $\mathcal{V}_{y}$ Hamiltonian. The red (light gray) curves are associated with hole states and the blue (dark gray) ones with particles states.

must be used to find the correct expression. The $x$ component of the probability currents in media I and III will be always the same in our problems and are found to be

$$
J_{I x}=\left[1-\left|r_{1}\right|^{2}-\left|r_{2}\right|^{2}\right] \cos \theta
$$

and

$$
J_{I I I x}=\left[\left|t_{1}\right|^{2}+\left|t_{2}\right|^{2}\right] \cos \theta .
$$

With these, we see that, for barrier problems

$$
J_{I x}=J_{I I I x} \Rightarrow 1=\left|r_{1}\right|^{2}+\left|r_{2}\right|^{2}+\left|t_{1}\right|^{2}+\left|t_{2}\right|^{2},
$$

and we recover the usual result. In step problems, we will have different coefficients, depending on the states involved in the scattering at the interface.

\section{SEMI-INFINITE REGIONS: STEP PROBLEM}

$$
\mathcal{V}_{y} \text { interaction }
$$

This problem is very much alike the usual quantum mechanical problem of transmission through a potential energy step smaller than the particle's energy. In Fig. 3 , we show the energetics of the problem and we easily see that for $k<\Delta, k$ being the energy of the incident particle, there will be no transmission, and hence we need the energy of the incident particle to be larger than $\Delta$. Moreover, conservation of energy and momentum along the $y$ direction demands that

$$
\begin{aligned}
k & =\sqrt{q^{2}+\Delta^{2}} \\
k \sin \theta & =q \sin \alpha .
\end{aligned}
$$

where $\alpha$ is the angle between the $y$ and $x$ components of the wavevector of the transmitted wave. The transmission can take place with any of the two degenerate states 
of positive energy, and, consequently, the boundary conditions at the interface leads us to the system

$$
\begin{aligned}
& \frac{1}{\sqrt{2}}\left(\begin{array}{c}
e^{-i \theta / 2} \\
e^{i \theta / 2} \\
0 \\
0
\end{array}\right)+\frac{r_{1}}{\sqrt{2}}\left(\begin{array}{c}
-i e^{i \theta / 2} \\
i e^{-i \theta / 2} \\
0 \\
0
\end{array}\right)+\frac{r_{2}}{\sqrt{2}}\left(\begin{array}{c}
0 \\
0 \\
i e^{-i \theta / 2} \\
-i e^{i \theta / 2}
\end{array}\right) \\
& =\frac{t_{1}}{\sqrt{2}}\left(\begin{array}{c}
A_{+} e^{-i \alpha / 2} \\
A_{+} e^{i \alpha / 2} \\
A_{-} e^{i \alpha / 2} \\
-A_{-} e^{-i \alpha / 2}
\end{array}\right)+\frac{t_{2}}{\sqrt{2}}\left(\begin{array}{c}
A_{-} e^{-i \alpha / 2} \\
-A_{-} e^{i \alpha / 2} \\
-A_{+} e^{i \alpha / 2} \\
-A_{+} e^{-i \alpha / 2}
\end{array}\right) . \quad(46)
\end{aligned}
$$

Its solutions are

$$
\begin{aligned}
& r_{1}=\frac{q \sin \alpha-k \sin \theta}{k+q \cos (\alpha+\theta)} \\
& r_{2}=-i \Delta \frac{\cos \theta}{k+q \cos (\alpha+\theta)} \\
& t_{1}=\sqrt{2 k(k+q)} \frac{\cos \theta \cos \left(\frac{\alpha+\theta}{2}\right)}{k+q \cos (\alpha+\theta)} \\
& t_{2}=i \sqrt{2 k(k-q)} \frac{\cos \theta \sin \left(\frac{\alpha+\theta}{2}\right)}{k+q \cos (\alpha+\theta)}
\end{aligned}
$$

which shows that there are reflection and transmission to all the energy degenerate states. Similar expressions for the transmission and reflection amplitudes and probabilities (which follow below) are always found when dealing with wave-function matching in graphene and have previously been derived in the literature [18, 19].

The evaluation of the probability current in medium II reveals that $J_{I I x}=\frac{q}{k}\left(\left|t_{1}\right|^{2}+\left|t_{2}\right|^{2}\right) \cos \alpha$. Hence, by (39) and 41),

$$
T=\left(\left|t_{1}\right|^{2}+\left|t_{2}\right|^{2}\right) \frac{q}{k} \frac{\cos \alpha}{\cos \theta} .
$$

We can use (44), the coefficients (47)-(50) and the definition of $\alpha=\tan ^{-1}\left(q_{y} / q_{x}\right)$, to express this transmission probability (TP) as a function of the given parameters of the disturbing potential and of the incident wave.

So we get

$$
T=\frac{2 \sqrt{1-\eta^{2}} \cos \alpha}{1+\sqrt{1-\eta^{2}} \cos \alpha},
$$

with the angle of emergence given by

$$
\alpha=\tan ^{-1}\left(\frac{\sin \theta}{\sqrt{\cos ^{2} \theta-\eta^{2}}}\right),
$$

where we defined $\eta \equiv \Delta / k$ (this procedure will be repeated throughout the whole paper in a more succinct fashion, unless some special warning is necessary).

One should be careful about some details of these solutions. First of all, one notices from (53) that there are situations in which, even if the energy of the particle is greater than the interaction energy, for large enough incidence angles, $\alpha$ will be complex, also leading to a complex TP, which has no physical meaning. This phenomenon is analogous to total reflection in electromagnetism. This happens because although in the transmission and reflection probabilities (TP and RP) expressions the transmission and reflection amplitudes appear only in squared moduli, the probability current also depends on the angles. Making the substitution of imaginary $q_{x}$ and $\alpha$ first in the wavefunctions and then proceeding with the calculation of $J_{I I x}$ reveals that actually $J_{I I x}=0$, as expected. This is the same as happens for scattering by step potentials in usual quantum mechanics when we deal with energies above and below the step energy.

In Fig. 4, we show the general behavior of the TP. Some interesting features of the problem arise now, as we see that state 2 only contributes to transmission about the extreme values of the allowed angles. As we raise the barrier in relation to the particle's energy, we see that the probability of transmission drops to zero, as expected. We also note that there is a focalization of the beam, with respect to the possible incidence angles which allow for transmission with non-zero probability. Looking at the behavior of the "angles of refraction", which characterize the direction of propagation of the wave-fronts of the spatial parts of the wavefunction, as a function of the angle of incidence, we see that for small values they are about the same, at least for low $\eta$. One should notice that these angles need not have actual relation to the direction of propagation of the probability current, since the spinors also depend on the momenta and this affects the mean value of the current operator (it is connected to the spinors through the $\vec{\sigma}$ matrices).

\section{$\mathcal{V}_{x}$ interaction}

The scheme of the dispersion relations for this physical situation is shown in Fig. 5.

We notice that there are two non-degenerate bands accessible to the incident particles (we will call these the states 1 and 2), which lead to particles moving with different momenta inside the medium, due to the conservation of energy. This will lead to two different angles $\alpha_{i}$, $i=1,2$, for the emergent particles on the right side of the interface, related to the angle of incidence $\theta$ by

$$
q_{i} \sin \alpha_{i}=k \sin \theta .
$$

There can also be two different cases, which will change the states accessible to the emergent particles, namely $k>\Delta$ and $k<\Delta$. These considerations lead to the following situations:

i) $k>\Delta$ In this case (Fig. 5 left), the accessible states in medium II are given by $\left|\chi_{++}\right\rangle$and $\left|\chi_{+-}\right\rangle$from equation (25) and we have

$$
k=q_{1}+\Delta=q_{2}-\Delta
$$



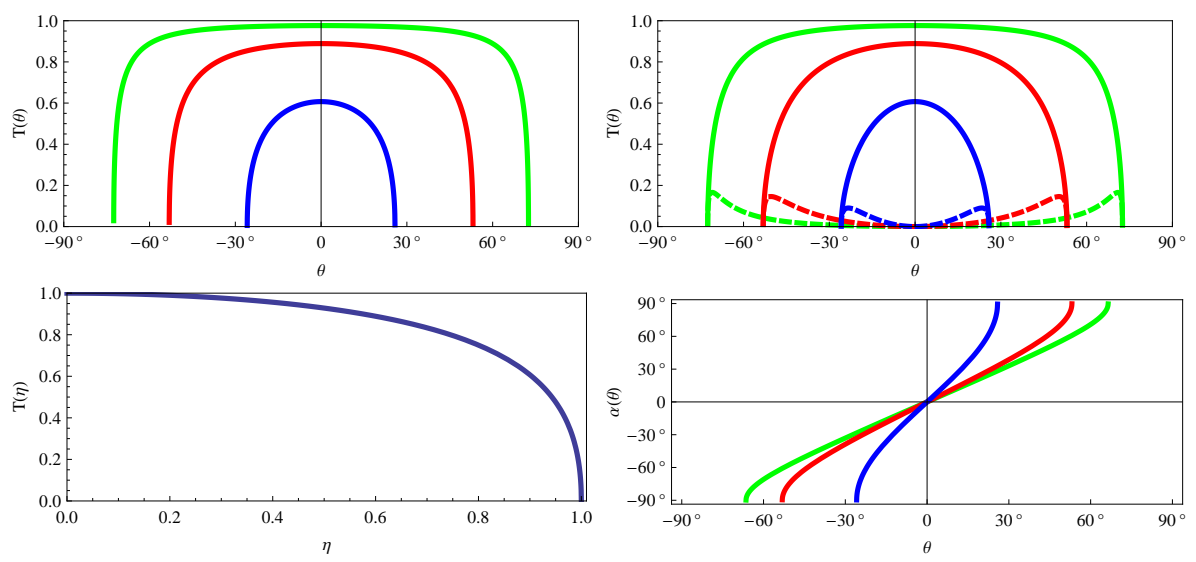

Figure 4: (color on-line) Behavior of the TP for the $\mathcal{V}_{y}$ step problem. In the upper and lower figures on the right, we have $\eta$ equals to $0.3,0.6$ and 0.9 , for the green (light gray), red (mid gray) and blue (dark gray) curves, respectively. (Upper left) Total TP as a function of the angle of incidence. (Upper right) We separate here the TP associated with states 1 (full line) and 2(dashed line). (Lower left) TP for normal incidence as a function of the ratio of the energies of interaction and incidence. It's important to notice that the contributions here come only from state 1. (Lower right) "Refraction angle" as a function of the incident angle for the same values of $\eta$ above.

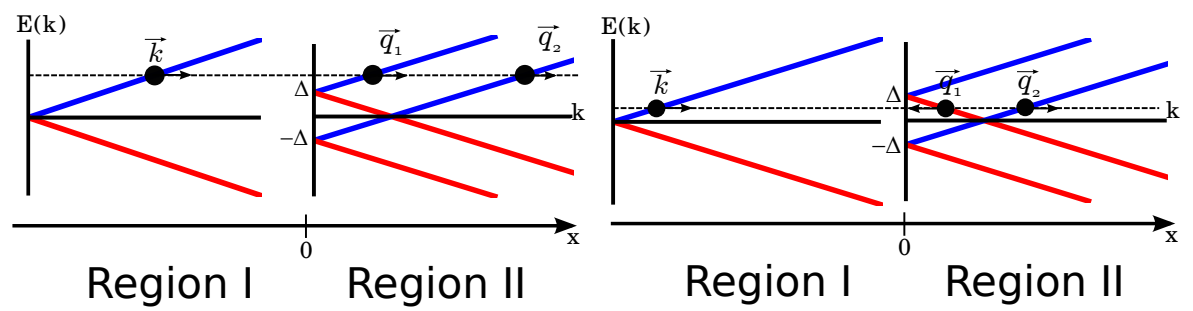

Figure 5: (color on-line) Scheme of the dispersion relation for the step transmission problem for Hamiltonian $\mathcal{V}_{x}$. The red (light gray) curves are associated with hole states and the blue (dark gray) ones with particles states. (left) Transmission for $k>\Delta$. (right) Transmission for $k<\Delta$.

and the boundary condition at the interface leads to

$$
\frac{1}{\sqrt{2}}\left(\begin{array}{c}
e^{-i \theta / 2} \\
e^{i \theta / 2} \\
0 \\
0
\end{array}\right)+\frac{r_{1}}{\sqrt{2}}\left(\begin{array}{c}
-i e^{i \theta / 2} \\
i e^{-i \theta / 2} \\
0 \\
0
\end{array}\right)+\frac{r_{2}}{\sqrt{2}}\left(\begin{array}{c}
0 \\
0 \\
i e^{-i \theta / 2} \\
-i e^{i \theta / 2}
\end{array}\right)=\frac{t_{1}}{2}\left(\begin{array}{c}
e^{-i \alpha_{1} / 2} \\
e^{i \alpha_{1} / 2} \\
e^{i \alpha_{1} / 2} \\
e^{-i \alpha_{1} / 2}
\end{array}\right)+\frac{t_{2}}{2}\left(\begin{array}{c}
e^{-i \alpha_{2} / 2} \\
e^{i \alpha_{2} / 2} \\
-e^{i \alpha_{2} / 2} \\
-e^{-i \alpha_{2} / 2}
\end{array}\right) .
$$

The solution of the linear system in this case is

$$
\begin{aligned}
& r_{1}=\frac{1}{2} \frac{\left[\sin \left(\frac{\alpha_{1}+\alpha_{2}}{2}\right)-\cos \left(\frac{\alpha_{1}-\alpha_{2}}{2}\right) \sin \theta\right]}{\cos \left(\frac{\alpha_{1}+\theta}{2}\right) \cos \left(\frac{\alpha_{2}+\theta}{2}\right)}, \\
& r_{2}=\frac{1}{2} \frac{\sin \left(\frac{\alpha_{1}-\alpha_{2}}{2}\right) \cos \theta}{\cos \left(\frac{\alpha_{1}+\theta}{2}\right) \sin \left(\frac{\alpha_{2}+\theta}{2}\right)}, \\
& t_{1}=\frac{\cos \theta}{\sqrt{2} \cos \left(\frac{\alpha_{1}+\theta}{2}\right)}, \\
& t_{2}=\frac{\cos \theta}{\sqrt{2} \cos \left(\frac{\alpha_{2}+\theta}{2}\right)} .
\end{aligned}
$$

The probability current in the $x$ direction is given by

$$
J_{I I x}=e\left(\left|t_{1}\right|^{2} \cos \alpha_{1}+\left|t_{2}\right|^{2} \cos \alpha_{2}\right),
$$

leading to the transmission coefficient

$$
T=\frac{\cos \alpha_{1}}{\cos \theta}\left|t_{1}\right|^{2}+\frac{\cos \alpha_{2}}{\cos \theta}\left|t_{2}\right|^{2} .
$$


Substituting the transmission amplitudes of the solution gives

$$
T=\cos \theta\left\{\frac{\cos \alpha_{1}}{1+\cos \left[\alpha_{1}+\theta\right]}+\frac{\cos \alpha_{2}}{1+\cos \left[\alpha_{2}+\theta\right]}\right\} .
$$

Proceeding in the same way as in the last section, we get

$$
\begin{aligned}
& \alpha_{1}=\tan ^{-1}\left(\frac{\sin \theta}{\sqrt{(1-\eta)^{2}-\sin ^{2} \theta}}\right) ; \\
& \alpha_{2}=\tan ^{-1}\left(\frac{\sin \theta}{\sqrt{(1+\eta)^{2}-\sin ^{2} \theta}}\right) ;
\end{aligned}
$$

with which, one can plot the behavior of the TP, analogously to what we have shown in Fig. 6 .

Conservation of the $y$ component of the particle's momentum would reveal that, in analogy to the classical electromagnetic case, particles of band 1 feel the medium
II "less refractive" and, consequently, are fully reflected. However, for particles of band 2, the medium is "more refractive" and there will always be transmission.

We notice that the main contribution to the total TP of states of band 1 (those subject to total reflection) comes mostly from small angles $\theta$, so the current must be almost normal to the interface if one expects to transmit these particles. Looking at the "angles of refraction" as a function of the angle of incidence, one can easily recognize which band is subject to total reflection.

ii) $k<\Delta$ In this case, we have the possibility of conversion of a particle state into a hole state, as shown in the right diagram of Fig. 5, and the conservation of energy gives us

$$
k=-q_{1}+\Delta=q_{2}-\Delta .
$$

The linear system to be solved involves now the states $\left|\chi_{-+}\right\rangle$and $\left|\chi_{+-}\right\rangle$, from (25) and (26) respectively, and reads

$$
\frac{1}{\sqrt{2}}\left(\begin{array}{c}
e^{-i \theta / 2} \\
e^{i \theta / 2} \\
0 \\
0
\end{array}\right)+\frac{r_{1}}{\sqrt{2}}\left(\begin{array}{c}
-i e^{i \theta / 2} \\
i e^{-i \theta / 2} \\
0 \\
0
\end{array}\right)+\frac{r_{2}}{\sqrt{2}}\left(\begin{array}{c}
0 \\
0 \\
i e^{-i \theta / 2} \\
-i e^{i \theta / 2}
\end{array}\right)=\frac{t_{1}}{2}\left(\begin{array}{c}
e^{-i \alpha_{1} / 2} \\
-e^{i \alpha_{1} / 2} \\
-e^{i \alpha_{1} / 2} \\
e^{-i \alpha_{1} / 2}
\end{array}\right)+\frac{t_{2}}{2}\left(\begin{array}{c}
e^{-i \alpha_{2} / 2} \\
e^{i \alpha_{2} / 2} \\
-e^{i \alpha_{2} / 2} \\
-e^{-i \alpha_{2} / 2}
\end{array}\right)
$$

Its solution is

$$
\begin{gathered}
r_{1}=-\frac{1}{2} \frac{\left[\cos \left(\frac{\alpha_{1}+\alpha_{2}}{2}\right)+\sin \left(\frac{\alpha_{1}-\alpha_{2}}{2}\right) \sin \theta\right]}{\sin \left(\frac{\alpha_{1}+\theta}{2}\right) \cos \left(\frac{\alpha_{2}+\theta}{2}\right)} \\
r_{2}=-\frac{1}{2} \frac{\cos \left(\frac{\alpha_{1}-\alpha_{2}}{2}\right) \cos \theta}{\sin \left(\frac{\alpha_{1}+\theta}{2}\right) \cos \left(\frac{\alpha_{2}+\theta}{2}\right)} \\
t_{1}=i \frac{\cos \theta}{\sqrt{2} \sin \left(\frac{\alpha_{1}+\theta}{2}\right)} \\
t_{2}=\frac{\cos \theta}{\sqrt{2} \sin \left(\frac{\alpha_{2}+\theta}{2}\right)},
\end{gathered}
$$

and the probability current and TP are given by

$$
\begin{aligned}
J_{I I x} & =e\left(-\left|t_{1}\right|^{2} \cos \alpha_{1}+\left|t_{2}\right|^{2} \cos \alpha_{2}\right), \\
T & =-\frac{\cos \alpha_{1}}{\cos \theta}\left|t_{1}\right|^{2}+\frac{\cos \alpha_{2}}{\cos \theta}\left|t_{2}\right|^{2},
\end{aligned}
$$

which, after proper substitution of the coefficients leads to

$$
T=\cos \theta\left\{-\frac{\cos \alpha_{1}}{1-\cos \left[\alpha_{1}+\theta\right]}+\frac{\cos \alpha_{2}}{1+\cos \left[\alpha_{2}+\theta\right]}\right\} .
$$

One can see from this expression the expected appearance of the Klein paradox, by looking at the denominator of this expression's first term in the case of normal incidence. The solution of this is the well-known argument that the hole-like particle must have an inverted momentum, so that it will continue traveling from left to right in the expected direction 9 . For such, we demand

$$
\begin{aligned}
& \alpha_{1}=\pi+\tan ^{-1}\left(\frac{\sin \theta}{\sqrt{(1-\eta)^{2}-\sin ^{2} \theta}}\right), \\
& \alpha_{2}=\tan ^{-1}\left(\frac{\sin \theta}{\sqrt{(1+\eta)^{2}-\sin ^{2} \theta}}\right) .
\end{aligned}
$$

We notice that, with these conventions for the angles, the expression 63 is actually valid independently of the energy of the particles and their interaction energy, but we will keep the solutions separated. The general behavior of the TP for this case is shown in Fig. 7.

We notice that as we keep raising the barrier (or lowering the particle's energy), the contributions of both bands tend to become equal. It is interesting to see that when $\eta=2$, particles of band 1 go through the barrier as if there was nothing there, independently of the angle of incidence. The behavior of the particle in band 1 as a function of $\eta>1$ is such that the transmission starts 

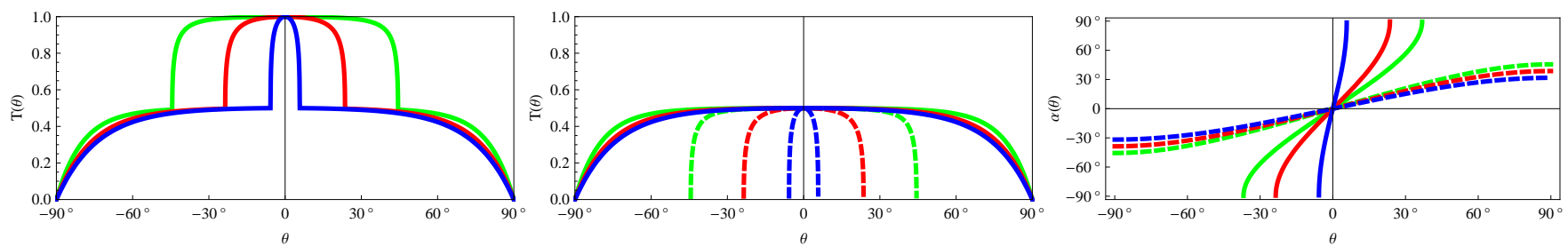

Figure 6: (color on-line) Behavior of the TP for the $\mathcal{V}_{x} k>\Delta$ step problem. The curves plotted are for $\eta$ equals to 0.3 , 0.6 and 0.9 corresponding to green (light gray), red (mid gray) and blue (dark gray) respectively. (Left) Total TP in function of the angle of incidence. (Middle) Contributions to the TP given by the separate states 1 (full line) and 2(dashed line). (Right) "Refraction angles" as a function of the incidence angle for the same values of $\eta$ above. Full lines correspond to $\alpha_{1}$ and dashed lines to $\alpha_{2}$.

around zero for $\eta \approx 1$ increases to uniform probability when $\eta=2$ and then tends to the same type of behavior of the particle in band 2 .

From the behavior of the angles $\alpha_{1}$ and $\alpha_{2}$ as a function of $\theta$ it is clear how, as we keep raising the barrier (or lowering the energies), the direction of propagation of the wave-fronts tend to become equal. For $\eta=2$ there is the explicit inversion of behavior for the particle in band 1 and it stops being subject to total reflection (notice that the rightmost plot of Fig. 7 is actually of $\alpha_{1}-\pi$ but, since the holes move contrary to their momenta, this is the actual direction of motion of the particle's wavefront).

We can use the results of these last two cases to prepare a quasi-particle current in the material in a definite quantum state or even in a known linear combination of states. For the normal incidence problem, we also see that there will be full transmission, independently of the relation of the energy of the particle to the strength of the interaction.

\section{FINITE REGIONS:BARRIER PROBLEMS}

Now we revisit the problems of the last sections for the case of a finite region of disturbed graphene. The mathematical developments proceed almost in the same way as before, the only difference being that we need to match the wavefunctions from medium I and medium II at $x=0$ and from medium II and medium III at $x=d, d$ being the width of the barrier, which will lead to a more complex linear system. Hence, we have the following situations.

\section{$\mathcal{V}_{y}$ interaction}

The matching of the wave function leads to the system

$$
\begin{aligned}
& \frac{1}{\sqrt{2}}\left(\begin{array}{c}
e^{-i \theta / 2} \\
e^{i \theta / 2} \\
0 \\
0
\end{array}\right)+\frac{r_{1}}{\sqrt{2}}\left(\begin{array}{c}
-i e^{i \theta / 2} \\
i e^{-i \theta / 2} \\
0 \\
0
\end{array}\right)+\frac{r_{2}}{\sqrt{2}}\left(\begin{array}{c}
0 \\
0 \\
i e^{-i \theta / 2} \\
-i e^{i \theta / 2}
\end{array}\right)= \\
& \frac{A_{1}}{\sqrt{2}}\left(\begin{array}{c}
A_{+} e^{-i \alpha / 2} \\
A_{+} e^{i \alpha / 2} \\
A_{-} e^{i \alpha / 2} \\
-A_{-} e^{-i \alpha / 2}
\end{array}\right)+\frac{A_{2}}{\sqrt{2}}\left(\begin{array}{c}
A_{-} e^{-i \alpha / 2} \\
-A_{-} e^{i \alpha / 2} \\
-A_{+} e^{i \alpha / 2} \\
-A_{+} e^{-i \alpha / 2}
\end{array}\right)+\frac{B_{1}}{\sqrt{2}}\left(\begin{array}{c}
-i A_{+} e^{i \alpha / 2} \\
i A_{+} e^{-i \alpha / 2} \\
i A_{-} e^{-i \alpha / 2} \\
i A_{-} e^{i \alpha / 2}
\end{array}\right)+\frac{B_{2}}{\sqrt{2}}\left(\begin{array}{c}
-i A_{-} e^{i \alpha / 2} \\
-i A_{-} e^{-i \alpha / 2} \\
-i A_{+} e^{-i \alpha / 2} \\
i A_{+} e^{i \alpha / 2}
\end{array}\right) \\
& \left.\frac{A_{1}}{\sqrt{2}}\left(\begin{array}{c}
A_{+} e^{-i \alpha / 2} \\
A_{+} e^{i \alpha / 2} \\
A_{-} e^{i \alpha / 2} \\
-A_{-} e^{-i \alpha / 2}
\end{array}\right)+\frac{A_{2}}{\sqrt{2}}\left(\begin{array}{c}
A_{-} e^{-i \alpha / 2} \\
-A_{-} e^{i \alpha / 2} \\
-A_{+} e^{i \alpha / 2} \\
-A_{+} e^{-i \alpha / 2}
\end{array}\right)\right] e^{i q_{x} d}+\left[\frac{B_{1}}{\sqrt{2}}\left(\begin{array}{c}
-i A_{+} e^{i \alpha / 2} \\
i A_{+} e^{-i \alpha / 2} \\
i A_{-} e^{-i \alpha / 2} \\
i A_{-} e^{i \alpha / 2}
\end{array}\right)+\frac{B_{2}}{\sqrt{2}}\left(\begin{array}{c}
-i A_{-} e^{i \alpha / 2} \\
-i A_{-} e^{-i \alpha / 2} \\
-i A_{+} e^{-i \alpha / 2} \\
i A_{+} e^{i \alpha / 2}
\end{array}\right)\right] e^{-i q_{x} d}= \\
& {\left[\frac{t_{1}}{\sqrt{2}}\left(\begin{array}{c}
e^{-i \theta / 2} \\
e^{i \theta / 2} \\
0 \\
0
\end{array}\right)+\frac{t_{2}}{\sqrt{2}}\left(\begin{array}{c}
0 \\
0 \\
e^{i \theta / 2} \\
e^{-i \theta / 2}
\end{array}\right)\right] e^{i k_{x} d}}
\end{aligned}
$$



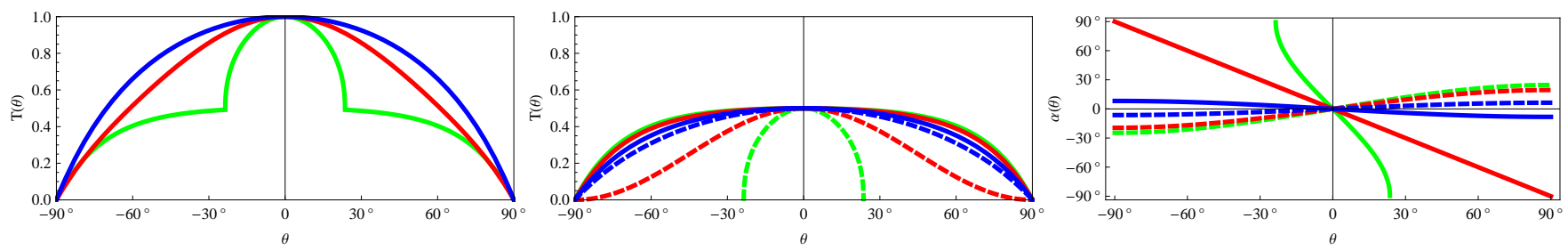

Figure 7: (color on-line) Behavior of the TP for the $\mathcal{V}_{x} k<\Delta$ step problem. The curves plotted are for $\eta$ equals to 1.4, 2.0 and 8.0 corresponding to green (light gray),red (mid gray) and blue (dark gray) respectively. (Left) Total TP in function of the angle of incidence. (middle) Contributions to the TP given by the separate states 1 (full lines) and 2(dashed lines). (right) behavior of $\alpha_{1}-\pi$ (full lines) and $\alpha_{2}$ (dashed lines) as a function of $\theta$.

Notice that in medium II, we can now have both transmitted and reflected waves, because of the presence of two interfaces. We can solve this to find the coefficients $A_{i}$ and $B_{i}$ in terms of $t_{i}$ and then solve a problem analogous to those of the last sections. The dependence of $J_{I I x}$ on the position variables can be easily seen to vanish, as one should expect, and the resulting transmission probability can be directly obtained,

$$
T=\left|t_{1}\right|^{2}+\left|t_{2}\right|^{2} .
$$

The expression for the coefficients are cumbersome, but we show the ones corresponding to the $t_{i}$ 's and $r_{i}$ 's. They are:

$$
\begin{aligned}
& r_{1}=i \frac{\sin \left(d q_{x}\right)(-q \sin \alpha+k \sin \theta)}{q \cos \left(d q_{x}\right) \cos \alpha \cos \theta+i \sin \left(d q_{x}\right)(k-q \sin \alpha \sin \theta)} \\
& r_{2}=-\frac{\sqrt{k^{2}-q^{2}} \cos \theta \sin \left(d q_{x}\right)}{q \cos \left(d q_{x}\right) \cos \alpha \cos \theta-i \sin \left(d q_{x}\right)(k-q \sin \alpha \sin \theta)} \\
& t_{1}=\frac{e^{-i d k_{x}} q \cos \alpha \cos \theta}{q \cos \left(d q_{x}\right) \cos \alpha \cos \theta-i \sin \left(d q_{x}\right)(k-q \sin \alpha \sin \theta)} \\
& t_{2}=0
\end{aligned}
$$

We are not able to write the expressions for the TPs in terms of the ratio of the incident particle's energy to the potential strength $\Delta$ anymore. In order to get an expression for the TPs, given only in terms of known variables, we now use

$$
\begin{aligned}
\alpha & =\tan ^{-1}\left(\frac{\sin \theta}{\sqrt{\cos ^{2} \theta-\left(\frac{\Delta}{k}\right)^{2}}}\right) ; \\
q_{x} & =k \sqrt{\cos ^{2} \theta-\left(\frac{\Delta}{k}\right)^{2}},
\end{aligned}
$$

finding

$$
T=\frac{\left(k^{2} \cos ^{2} \theta-\Delta^{2}\right)}{k^{2} \cos ^{2} \theta-\Delta^{2} \cos ^{2}\left(d \sqrt{k^{2} \cos ^{2} \theta-\Delta^{2}}\right)}
$$

The behavior of the TP is shown in Fig. 8. Since the emergent particles come out in a medium equal to the one from which they enter, we do not need to consider the incident and emergent angles, for they are the same. We also have the situation of total reflection in this case although, due to tunneling, the kinky behavior of the TP we had in the step problems is not apparent any more.

One should notice the oscillatory behavior generated by the self interference of the wave reflected inside the medium II (Fabry-Perot like interferences). In the middle plot, we realize that these oscillations are affected mainly by the width of the barrier. We also notice that raising the relative value of $\Delta$ over $k$ causes again a focalization of the beam and gives rise to a resonant phenomenon of full TP.

These resonant points can be easily analyzed. The TP for $\theta=0$ reduces to

$$
\left.T\right|_{\theta=0}=\frac{k^{2}-\Delta^{2}}{k^{2}-\Delta^{2} \cos ^{2}(d q)},
$$

whose maxima are seen to be given by

$$
d q_{n}=n \pi \Rightarrow k_{n}=\sqrt{\left(\frac{n \pi}{d}\right)^{2}+\Delta^{2}},
$$

which resembles the energy of massive relativistic particles in a box. Expanding $t_{1}(\theta=0)$ for $q$ around $n \pi / d$, we also get

$$
t_{1}(\theta=0) \approx \pm i \frac{\Gamma_{n} / 2}{k-k_{n}+i \Gamma_{n} / 2},
$$

where the $k_{n}^{\prime} s$ are, as above, the energy levels of virtual bound states whose inverse life-time (decay rates) are given by

$$
\Gamma_{n}=\left.2\left(\frac{k}{q}\left(\frac{d q}{d k}\right)\right)\right|_{q=\frac{n \pi}{d}}=2\left(1+\left(\frac{\Delta d}{n \pi}\right)^{2}\right)^{-1} .
$$

Similar life-times should appear for different values of the incident angle. We see that if, for fixed $n$ and $\Delta$, the barrier width gets larger, the particle life-time also increases. This is an expected result since the mean number of times the particle should reflect back and forth does not change and neither does its velocity, so the time spent inside the barrier should be longer if it is larger. If the interaction is stronger, i.e., the barrier is "higher", one expect the 
particle's quantum behavior to be more important and therefore, even if the particle's energy is above the barrier, it will spend "more time" inside it and we expect a longer life-time.

If in the normal incidence TP (84), both the interaction $\Delta$ and the energy $k$ are small, we have, up to second order in $k^{2}$ and $\Delta^{2}$,

$$
\left.T\right|_{\theta=0} \approx \frac{k^{2}-\Delta^{2}}{k^{2}-\Delta^{2}+\Delta^{2} d^{2}\left(k^{2}-\Delta^{2}\right)} .
$$

Decreasing the energy to arbitrarily low values leads to

$$
\left.T\right|_{\theta=0} \approx \frac{1}{1+d^{2} \Delta^{2}}
$$

Hence, if the interaction energy is low enough (or even if the barrier width is small enough, as can be easily checked), it is possible to have transmission for arbitrarily low energies, even if smaller than $\Delta$. This is a phenomenon analogous to tunneling in usual quantum mechanics barrier problems. The wavefunction component dependent on the $x$ direction becomes a real exponential, which can in some situations penetrate in the other medium.

Notice that the transmission amplitude for states $2\left(t_{2}\right)$ is identically zero. This means that there can be no flipping of the pseudo-spin due to the $\mathcal{V}_{y}$ coupling Hamiltonian. The situation is different for the $\mathcal{V}_{x}$ interaction, as will be shown in the next sections.

$\mathcal{V}_{x}$ interaction

i) $k>\Delta$ The linear system in this particular case is now

$$
\begin{aligned}
& \frac{1}{\sqrt{2}}\left(\begin{array}{c}
e^{-i \theta / 2} \\
e^{i \theta / 2} \\
0 \\
0
\end{array}\right)+\frac{r_{1}}{\sqrt{2}}\left(\begin{array}{c}
-i e^{i \theta / 2} \\
i e^{-i \theta / 2} \\
0 \\
0
\end{array}\right)+\frac{r_{2}}{\sqrt{2}}\left(\begin{array}{c}
0 \\
0 \\
i e^{-i \theta / 2} \\
-i e^{i \theta / 2}
\end{array}\right)= \\
& \frac{A_{1}}{2}\left(\begin{array}{c}
e^{-i \alpha_{1} / 2} \\
e^{i \alpha_{1} / 2} \\
e^{i \alpha_{1} / 2} \\
e^{-i \alpha_{1} / 2}
\end{array}\right)+\frac{B_{2}}{2}\left(\begin{array}{c}
e^{-i \alpha_{2} / 2} \\
e^{i \alpha_{2} / 2} \\
-e^{i \alpha_{2} / 2} \\
-e^{-i \alpha_{2} / 2}
\end{array}\right)+\frac{B_{1}}{2}\left(\begin{array}{c}
-i e^{i \alpha_{1} / 2} \\
i e^{-i \alpha_{1} / 2} \\
i e^{-i \alpha_{1} / 2} \\
i e^{i \alpha_{1} / 2}
\end{array}\right)+\frac{B_{2}}{2}\left(\begin{array}{c}
-i e^{i \alpha_{2} / 2} \\
-i e^{-i \alpha_{2} / 2} \\
-i e^{-i \alpha_{2} / 2} \\
i e^{i \alpha_{2} / 2}
\end{array}\right) \\
& \frac{A_{1}}{2}\left(\begin{array}{c}
e^{-i \alpha_{1} / 2} \\
e^{i \alpha_{1} / 2} \\
e^{i \alpha_{1} / 2} \\
e^{-i \alpha_{1} / 2}
\end{array}\right) e^{i q_{1 x} d}+\frac{B_{2}}{2}\left(\begin{array}{c}
e^{-i \alpha_{2} / 2} \\
e^{i \alpha_{2} / 2} \\
-e^{i \alpha_{2} / 2} \\
-e^{-i \alpha_{2} / 2}
\end{array}\right) e^{i q_{2 x} d}+\frac{B_{1}}{2}\left(\begin{array}{c}
-i e^{i \alpha_{1} / 2} \\
i e^{-i \alpha_{1} / 2} \\
i e^{-i \alpha_{1} / 2} \\
i e^{i \alpha_{1} / 2}
\end{array}\right) e^{-i q_{1 x} d}+\frac{B_{2}}{2}\left(\begin{array}{c}
-i e^{i \alpha_{2} / 2} \\
-i e^{-i \alpha_{2} / 2} \\
-i e^{-i \alpha_{2} / 2} \\
i e^{i \alpha_{2} / 2}
\end{array}\right) e^{-i q_{2 x} d}= \\
& {\left[\frac{t_{1}}{\sqrt{2}}\left(\begin{array}{c}
e^{-i \theta / 2} \\
e^{i \theta / 2} \\
0 \\
0
\end{array}\right)+\frac{t_{2}}{\sqrt{2}}\left(\begin{array}{c}
0 \\
0 \\
e^{i \theta / 2} \\
e^{-i \theta / 2}
\end{array}\right)\right] e^{i k_{x} d} .}
\end{aligned}
$$

The expressions for the solutions in this case are even more cumbersome than in the previous case, although they still resemble the expressions presented in the last section for this particular situation. We will show only the different behavior of the TP patterns related to them (Fig. 9). The angles $\alpha_{1}$ and $\alpha_{2}$ and the $x$ components of the momenta of states 1 and 2 are the same of those of the corresponding case of the previous section. We see that, for small angles, the resonant phenomena induced by the finite barrier is irrelevant. There is some selection of states for larger angles and only states of type 2 contribute to the central region. For the normal incidence, we also have a perfectly resonant behavior between the two TPs (which cause the total transmission be always equal to 1), as a function of the width of the barrier, which can be used to select the different states. There is no resonance for fixed width and variable energies of incident particles. 

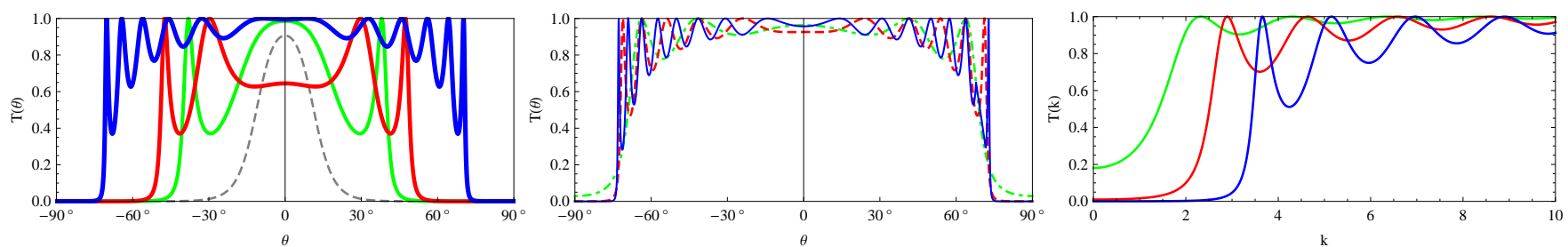

Figure 8: (color on-line) Behavior of the TP for the $\mathcal{V}_{y}$ barrier problem. Since $t_{2}=0$, we only plot the total TP. On the left we have $d=2.0$ and $\Delta=3.0$ fixed and vary the energy $k$ of the particles as 3.35 (gray, dashed light gray), 4.3 (green, full line light gray), 5.0 (red, mid gray) and 10.0 (blue, dark gray). In the middle, we fix $\Delta=4.45$ and $k=9.0$ and vary the width $d$ through 1.0(dashed green, light gray) , 2.0(dashed red, mid gray) and 3.0(full line blue, dark gray). On the right, we plot the normal TP as a function of $k$ for $d=1.5$ and $\Delta$ equals to 1.0 (green, light gray), 2.0 (red, mid gray), 3.0 (blue, dark gray).
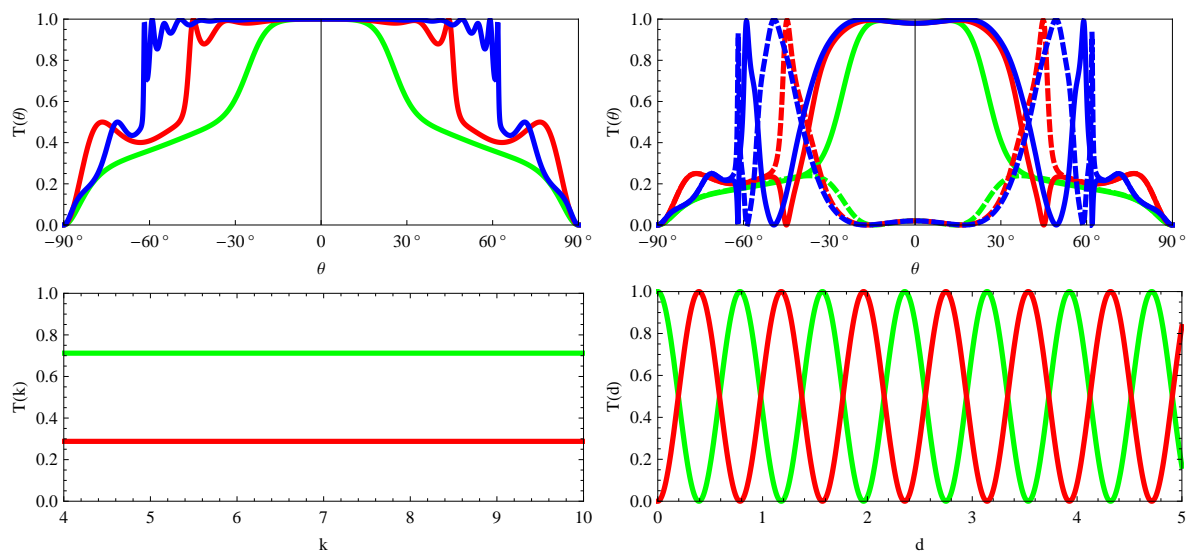

Figure 9: (color online) Behavior of the TP for the $\mathcal{V}_{x} k>\Delta$ barrier problem. (upper left) We have $d=3.0$ and $\Delta=1.0$ fixed and vary the energy $k$ of the particles as 2.0 (green, light gray), 4.0 (red, mid gray) and 9.0 (blue, dark gray). (upper right) We separate here the contributions to the total TP from the states 1 (full lines) and 2 (dashed lines). (Lower left) Contributions to the normal incidence given by state 1 (green, light gray) and state 2 (red, dark gray), for $\Delta=4.0$ and the same width of the barrier. (Lower Right) Contributions to the normal incidence given by state 1 (green, light gray) and state 2 (red, dark gray), for $\Delta=4.0$ and $k=5$, for a varying width of the barrier.

ii) $k<\Delta$ The system will change only through the transmitted state 1 within region II and reads

$$
\begin{gathered}
\frac{1}{\sqrt{2}}\left(\begin{array}{c}
e^{-i \theta / 2} \\
e^{i \theta / 2} \\
0 \\
0
\end{array}\right)+\frac{r_{1}}{\sqrt{2}}\left(\begin{array}{c}
-i e^{i \theta / 2} \\
i e^{-i \theta / 2} \\
0 \\
0
\end{array}\right)+\frac{r_{2}}{\sqrt{2}}\left(\begin{array}{c}
0 \\
0 \\
i e^{-i \theta / 2} \\
-i e^{i \theta / 2}
\end{array}\right)= \\
\frac{A_{1}}{2}\left(\begin{array}{c}
e^{-i \alpha_{1} / 2} \\
-e^{i \alpha_{1} / 2} \\
-e^{i \alpha_{1} / 2} \\
e^{-i \alpha_{1} / 2}
\end{array}\right)+\frac{B_{2}}{2}\left(\begin{array}{c}
e^{-i \alpha_{2} / 2} \\
e^{i \alpha_{2} / 2} \\
-e^{i \alpha_{2} / 2} \\
-e^{-i \alpha_{2} / 2}
\end{array}\right)+\frac{B_{1}}{2}\left(\begin{array}{c}
-i e^{i \alpha_{1} / 2} \\
-i e^{-i \alpha_{1} / 2} \\
-i e^{-i \alpha_{1} / 2} \\
-i e^{i \alpha_{1} / 2}
\end{array}\right)+\frac{B_{2}}{2}\left(\begin{array}{c}
-i e^{i \alpha_{2} / 2} \\
-i e^{-i \alpha_{2} / 2} \\
-i e^{-i \alpha_{2} / 2} \\
i e^{i \alpha_{2} / 2}
\end{array}\right) ; \\
\frac{A_{1}}{2}\left(\begin{array}{c}
e^{-i \alpha_{1} / 2} \\
-e^{i \alpha_{1} / 2} \\
-e^{i \alpha_{1} / 2} \\
e^{-i \alpha_{1} / 2}
\end{array}\right) e^{i q_{1 x} d}+\frac{B_{2}}{2}\left(\begin{array}{c}
e^{-i \alpha_{2} / 2} \\
e^{i \alpha_{2} / 2} \\
-e^{i \alpha_{2} / 2} \\
-e^{-i \alpha_{2} / 2}
\end{array}\right) e^{i q_{2 x} d}+\frac{B_{1}}{2}\left(\begin{array}{c}
-i e^{i \alpha_{1} / 2} \\
-i e^{-i \alpha_{1} / 2} \\
-i e^{-i \alpha_{1} / 2} \\
-i e^{i \alpha_{1} / 2}
\end{array}\right) e^{-i q_{1 x} d}+\frac{B_{2}}{2}\left(\begin{array}{c}
-i e^{i \alpha_{2} / 2} \\
-i e^{-i \alpha_{2} / 2} \\
-i e^{-i \alpha_{2} / 2} \\
i e^{i \alpha_{2} / 2}
\end{array}\right) e^{-i q_{2 x} d}=
\end{gathered}
$$




$$
\left[\frac{t_{1}}{\sqrt{2}}\left(\begin{array}{c}
e^{-i \theta / 2} \\
e^{i \theta / 2} \\
0 \\
0
\end{array}\right)+\frac{t_{2}}{\sqrt{2}}\left(\begin{array}{c}
0 \\
0 \\
e^{i \theta / 2} \\
e^{-i \theta / 2}
\end{array}\right)\right] e^{i k_{x} d},
$$

whose solution gives the TP plotted in Fig. 10. Here again we notice the already familiar behavior of selection of states 2 only in the central region. We notice that the solutions behave just like a continuation of the $k>\Delta$ problem and we have no energy resonances for the normal transmission problem. For small energy we have a transmission probability with poorly defined peaks, although if we look at the separate contributions of the two states, we see that they are such that the maxima and minima are opposite, except for normal transmission. Changing the size of the barrier, once again, induces resonances.

\section{CONDUCTANCE AND THE LANDAUER FORMALISM}

We will try to use now the results obtained above to access a measurable quantity, namely, the conductance of the material due to the presence of the barriers. We understand that our solution here might be an oversimplification, since we are not dealing with disorder, finite size effects, contacts, etc. Nevertheless, we believe that this might give us some insight on the usefulness of results like ours. We shall use a reasoning based on the Landauer formalism. The total transmitted current through our sample should be given by

$$
I \approx\left(\mu_{1}-\mu_{2}\right) \int d E \sum_{i} \mathcal{D}_{1}^{i}(E) J_{t}^{i}(E)\left[-\frac{\partial f_{0}(E)}{\partial E}\right]
$$

where $\mathcal{D}_{1}^{i}(E)$ is the one dimensional density of states per unit length for channel $i, J_{t}^{i}(E)$ is the total transmitted current through our barrier through channel $i$ and the sum is over all the channels. $\mu_{1}$ and $\mu_{2}$ are the respective chemical potentials of two particle reservoirs thorough which the electrons will flow and $f_{0}$ is the Fermi distribution with null chemical potential.

This leads to

$$
\begin{gathered}
\frac{I}{\left(\mu_{1}-\mu_{2}\right) e}=\frac{I}{\Delta \phi} \\
G \approx e \int d E \sum_{i} \mathcal{D}_{1}^{i}(E) J_{t}^{i}(E)\left[-\frac{\partial f_{0}(E)}{\partial E}\right],
\end{gathered}
$$

where $\Delta \phi$ is the potential difference and $G$ is the conductance between the reservoirs.

The channels $i$ are given by the discrete values that $k_{y}$ would assume in a finite sized sample, in such a way that the dispersion relation is to be given by (putting back the $\hbar$ 's)

$$
\begin{aligned}
E^{2} & =p^{2} \Rightarrow E^{2}=p_{x}^{2}+\hbar^{2}(i \pi / L)^{2} \\
p_{x}^{2} & =E^{2}-\hbar^{2}(i \pi / L)^{2} \\
p_{i}^{2} & \equiv E^{2}-\epsilon_{i}^{2},
\end{aligned}
$$

where $\epsilon_{i}$ takes the value for $p_{y}, p_{i}$ the value for $p_{x}$ and $i$ are integer numbers. Notice that $p_{i}$ would give the energy for a Dirac particle moving in graphene in one dimension at the channel $i$. The total current we are dealing with here, $J_{t}^{i}(E)$, is normal to the barrier, the possibilities of different incident angles is absorbed in the channels as we make clear below.

Therefore, with this prescription, the transmission probability for the $\mathcal{V}_{y}$ interaction is given by

$$
\begin{aligned}
T & =\frac{\left(E^{2} \cos ^{2} \theta-\Delta^{2}\right)}{E^{2} \cos ^{2} \theta-\Delta^{2} \cos ^{2}\left(d \sqrt{E^{2} \cos ^{2} \theta-\Delta^{2}}\right)} \\
& =\frac{\left(E^{2}-p_{y}^{2}-\Delta^{2}\right)}{E^{2}-p_{y}^{2}-\Delta^{2} \cos ^{2}\left(d \sqrt{E^{2}-p_{y}^{2}-\Delta^{2}}\right)} \\
\Rightarrow T^{i}(E) & =\frac{\left(E^{2}-\epsilon_{i}^{2}-\Delta^{2}\right)}{E^{2}-\epsilon_{i}^{2}-\Delta^{2} \cos ^{2}\left(d \sqrt{E^{2}-\epsilon_{i}^{2}-\Delta^{2}}\right)} \\
\text { or } T\left(E\left(p_{i}\right)\right) & =\frac{\left(p_{i}^{2}-\Delta^{2}\right)}{p_{i}^{2}-\Delta^{2} \cos ^{2}\left(d \sqrt{p_{i}^{2}-\Delta^{2}}\right)},
\end{aligned}
$$

where $E\left(p_{i}\right)=p_{i}$, which makes explicit our argument that the transmissions are 1D-like, normal to the barrier, and separated in different channels.

Now let's analyze the expression for the current. We can write,

$$
\begin{aligned}
\sum_{i} \mathcal{D}_{1}^{i}(E) J_{t}^{i}(E) & =\sum_{i} J_{t}^{i}(E) \frac{1}{L} \sum_{p_{i}} \delta\left(E-E\left(p_{i}\right)\right) \\
& =\frac{1}{L} \sum_{p_{i}, i} \delta\left(E-E\left(p_{i}\right)\right) J_{t}^{i}(E),(97)
\end{aligned}
$$

and using the results of sec. IV for the transmission direction only, $J_{t}^{i}(E) \equiv e T^{i}(E)\left|v_{x}\left(p_{i}\right)\right|=e T\left(E\left(p_{i}\right)\right)\left|v_{x}\left(p_{i}\right)\right|$ ( the transmission is assumed to be along the $x$ direction and the modulus is present to guarantee that only the positive $x$ direction is to be considered), one has

$$
\sum_{i} \mathcal{D}_{1}^{i}(E) J_{t}^{i}(E)=
$$



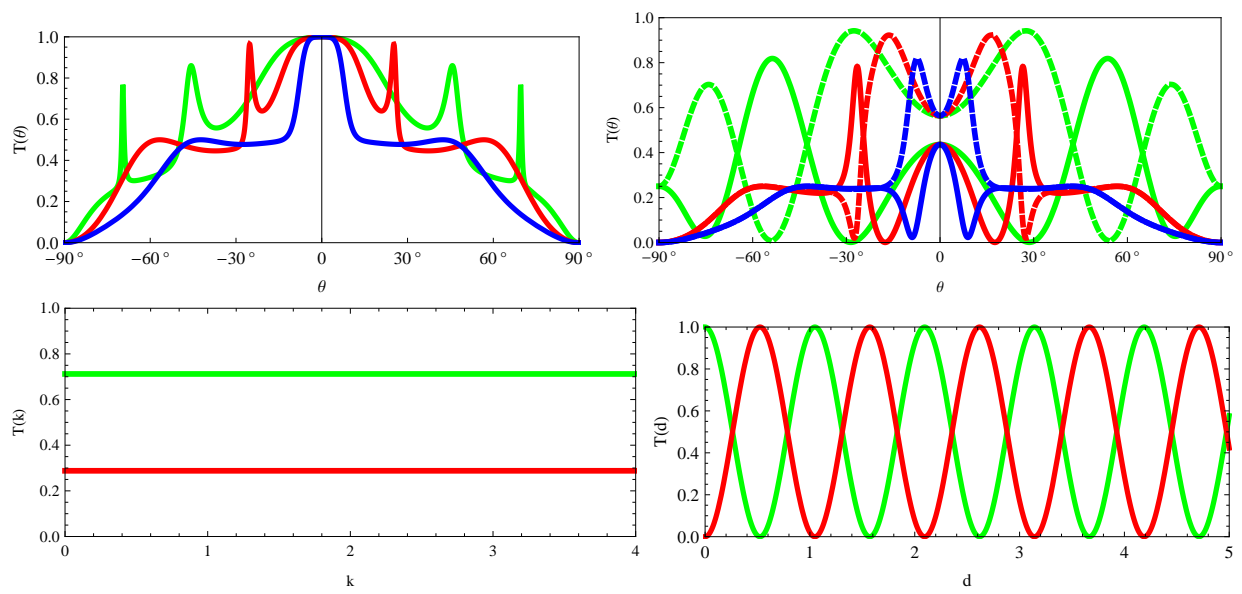

Figure 10: (color online) Behavior of the TP for the $\mathcal{V}_{x} k<\Delta$ barrier problem . (Upper left) We have $d=3.0$ and $\Delta=6.0$ fixed and vary the energy $k$ of the particles as 3.0 (green, full line light gray), 4.0 (red, mid gray) and 5.0 (blue, dark gray). In the middle, we separate the contributions to the total TP from the states 1 (full lines) and 2 (dashed lines) for the same values of $d, \Delta, k$. (Lower left) The figure shows the contributions to the normal incidence given by state 1 (green, light gray) and state 2 (red, dark gray), for $\Delta=4.0$. Notice the continuation of this last graphic in comparison to the lower left graphic in figure 9. (Lower right) Contributions to the normal incidence given by state 1 (green, light gray) and state 2 (red, dark gray), for $\Delta=4.0$ and $k=3$, for a varying width of the barrier.

$$
\begin{aligned}
& =e \frac{1}{L} \sum_{p_{i}, i} \delta\left(E-E\left(p_{i}\right)\right) T\left(E\left(p_{i}\right)\right)\left|v_{x}\left(p_{i}\right)\right| \\
& =e \frac{1}{L} \sum_{p_{i}, i} \delta\left(E-E\left(p_{i}\right)\right) T(E)\left|v_{x}\left(p_{i}\right)\right| \\
& =e \frac{1}{L} T(E) \sum_{p_{i}, i} \delta\left(E-E^{i}\left(p_{i}\right)\right)\left|v_{x}\left(p_{i}\right)\right|,
\end{aligned}
$$

where $L$ is the length of the sample. Now we can stop looking at the values of $p_{y}$ as different modes and sum over all the values of the vector $\mathbf{k}$ ( in two dimensions) obtaining

$$
\begin{aligned}
\sum_{i} \mathcal{D}_{1}^{i}(E) J_{t}^{i}(E) & =e \frac{1}{L} T(E) \sum_{p_{i}, i} \delta\left(E-E\left(p_{i}\right)\right)\left|v_{x}\left(p_{i}\right)\right| \\
& =\frac{e}{\pi \hbar} T(E) \sum_{\mathbf{p}} \delta(E-E(\mathbf{k})) \frac{\pi \hbar}{L}\left|v_{x}(\mathbf{p})\right| \\
& =\frac{e}{\pi \hbar} T(E) \underbrace{\sum_{\mathbf{p}} \delta(E-E(\mathbf{p})) \frac{\pi \hbar}{L}\left|v_{x}(\mathbf{p})\right|}_{\equiv M(E)} \\
& =\frac{e}{\pi \hbar} T(E) M(E) .
\end{aligned}
$$

The function $M(E)$ defines our number of transverse modes. It can be calculated by the usual method, transforming the sum in an integral and remembering to count the spin degeneracy. Valley degeneracy should not be included because the electrons are chosen in a very well defined valley, otherwise we should add another factor of 2 multiplying the final expression. It gives, for a sample of width $W$

$$
M(E)=\frac{2 W}{\pi \hbar}|E|
$$

or, putting back the Fermi velocity(remember that in our units were such that $v_{F}=1$ )

$$
M(E)=\frac{2 W}{\pi \hbar v_{F}}|E| .
$$

We can easily determine, now, the value of the conductance due to our barrier. For zero temperature (in which case the integral is trivial) we get,

$$
G=\frac{e^{2}}{\pi \hbar} T\left(E_{F}\right) \frac{2 W}{\pi \hbar v_{F}} E_{F}
$$

(notice that the units are correct).

Hence, we show that the conductance is expected to be proportional to the TP in the normal direction with the energy set at $E_{F}$. As we have shown and analyzed in the last section the behavior of this TP we will neither bother writing the explicit expressions nor going through the analysis of the graphic representation once again. The only comment we believe it deserves is to notice that for the $\mathcal{V}_{x}$ case we expect to be able to choose the contributions to the conductance for each different cone, as a function of the width of the barrier. We also expect oscillatory behavior of the conductance as we vary the energy in the case $\mathcal{V}_{y}$.

\section{CONCLUSIONS}

We analyzed the problem of electronic transmission in graphene through interfaces between regions in which quasi-particles belonging to different valleys (Dirac cones) interact or not. The relevant Hamiltonians we have employed are seen to be able to either create a gap 
in the quasi-particle spectrum or shift the Dirac cones with respect to each other. In the latter case, hole states become available to positive energy incident particles and the Klein paradox arises.

The behavior of the TP indicate that, for both Hamiltonians, in barrier and step like problems, there is focalization of the incident beam. In barrier problems, due to the behavior of the wavefunctions in each different region, we see that it should be possible to have situations in which we can enter the system with particles with momenta around one of the Dirac cones and come out with a superposition of electronic states about both cones. We could also act in the reverse way and filter states from one specific cone out of a general superposition of electronic states involving different valleys. If systems in which the electron-electron interactions we proposed can be isolated, this physical phenomenon could be useful for the development of "valleytronics", without dealing with edge modes [20, 21].

Another possibility is to use this kind of transmission to entangle a pair of originally separable electronic states belonging to different cones. This would be very useful if one wishes to employ a graphene sheet in the development of quantum processors.

In order to observe these effects, one should measure the contact resistance of small graphene samples placed on appropriate substrates which would induce the desired electron-electron interaction. Some considerations towards this were given in the last section, where we showed that the behavior of the conductance, at very low temperatures, is expected to be proportional to the $\mathrm{TP}$ normal to the given barrier.

Finally, a word of caution about the Hamiltonians we have used to induce the coupling between different valleys. Although we have appealed to general arguments to propose the phenomenological forms we have employed, we do not yet know of any microscopic mechanism to deduce them. Nevertheless, we believe that they can indeed be obtained from a more microscopic approach and shall be investigating this possibility in the near future.

\section{Acknowledgments}

P. L. e S. Lopes would like to thank "Fundação de Amparo à Pesquisa no Estado de São Paulo" (FAPESP, Brazil) for financial support under Grant No. 2009/18336-0 and A.O.C. acknolwedges financial support from FAPESP and CNPq through the Instituto Nacional de Ciência e Tecnologia em Informação Quântica (INCTIQ).
[1] Castro Neto, A. H.,Guinea, F., Peres, N. M. R., Novoselov, K. S. and Geim A. K., Rev. Mod. Phys. 81, 109 (2009);

[2] Peres, N. M. R., 2010, Rev. Mod. Phys. 82, 2673;

[3] V. M. Pereira and A. H. Castro Neto, Phys. Rev. Lett. 103, 046801 (2009);

[4] T. Ando and T. Nakanishi and R. Saito, J. Phys. Soc. Jpn. 67 (1998), 2857;

[5] E. McCann, K. Kechedzhi, V. I. Fal'ko, H. Suzuura, T. Ando and B. L. Altshuler, et al, Phys. Rev. Lett. 97 (2006), 146805;

[6] J. E. Drut and Timo A. Lahde, Phys. Rev. B 79 (2009), 165425;

[7] A. H. Castro Neto, Physics 2 (2009), 30;

[8] S. Ryu and C. Mudry and C.-Y. Hou and C. Chamon, Phys. Rev. B 80 (2009), 205319;

[9] A. Calogeracos and N. Dombey, Contemporary Physics 40 (1999);

[10] V. N. Kotov, B. Uchoa, V. M. Peirera, A. H. Castro Neto, and F. Guinea, 2010, preprint, arXiv:1012.3484,
[11] S. Das Sarma, S. Adam, E. H. Hwang, E. Rossi, 2010, preprint , arXiv:1003.4731.

[12] S. Das Sarma E. H. Hwang and W. Tse, Phys. Rev. B 75 (2007), 121406;

[13] J. Alicea and Fisher P. A., Phys. Rev. B 74 (2006), 075422;

[14] Y. Nazarov, Quantum Transport,Cambridge UP, Cambridge, (2009);

[15] Y. Imry, Introduction to Mesoscopic Systems, Oxford UP, Oxford, (1997);

[16] Imry Y., Landauer R., 1999, Rev. Mod. Phys. 71, S306;

[17] Beenakker, C. W., 2008, Rev. Mod. Phys. 80, 1337;

[18] Katsnelson, M. I., K. S. Novoselov, and A. K. Geim, 2006, Nat. Phys. 2, 620;

[19] Morpurgo, A. F., F. Guinea, 2006, PRL 97, 196804;

[20] Rycerz A., Tworzydlo J., Beenakker C. W., 2007, Nat. Phys. 3, 172;

[21] Garcia-Pomar J. L., Cortijo A., Nieto-Vesperinas M., 2008, PRL 100, 236801; 